\title{
RECYCLED CELLULOSE POLYPROPYLENE COMPOSITE FEEDSTOCKS FOR MATERIAL EXTRUSION ADDITIVE MANUFACTURING
}

Nicole E. Zander ${ }^{*}$, Jay H. Park², Zachary R. Boelter ${ }^{l}$ and Margaret A. Gillan ${ }^{3}$

${ }^{1}$ U.S. Army Research Laboratory, Weapons and Materials Research Directorate, Aberdeen Proving Ground, Aberdeen, MD

${ }^{2}$ University of Massachusetts Lowell, Francis College of Engineering, Lowell, MA

${ }^{3}$ Rowan University, Henry M. Rowan College of Engineering, Glassboro, NJ

*email: nicole.e.zander.civ@mail.mil; telephone: 410-306-1965 


\section{Supporting Information}
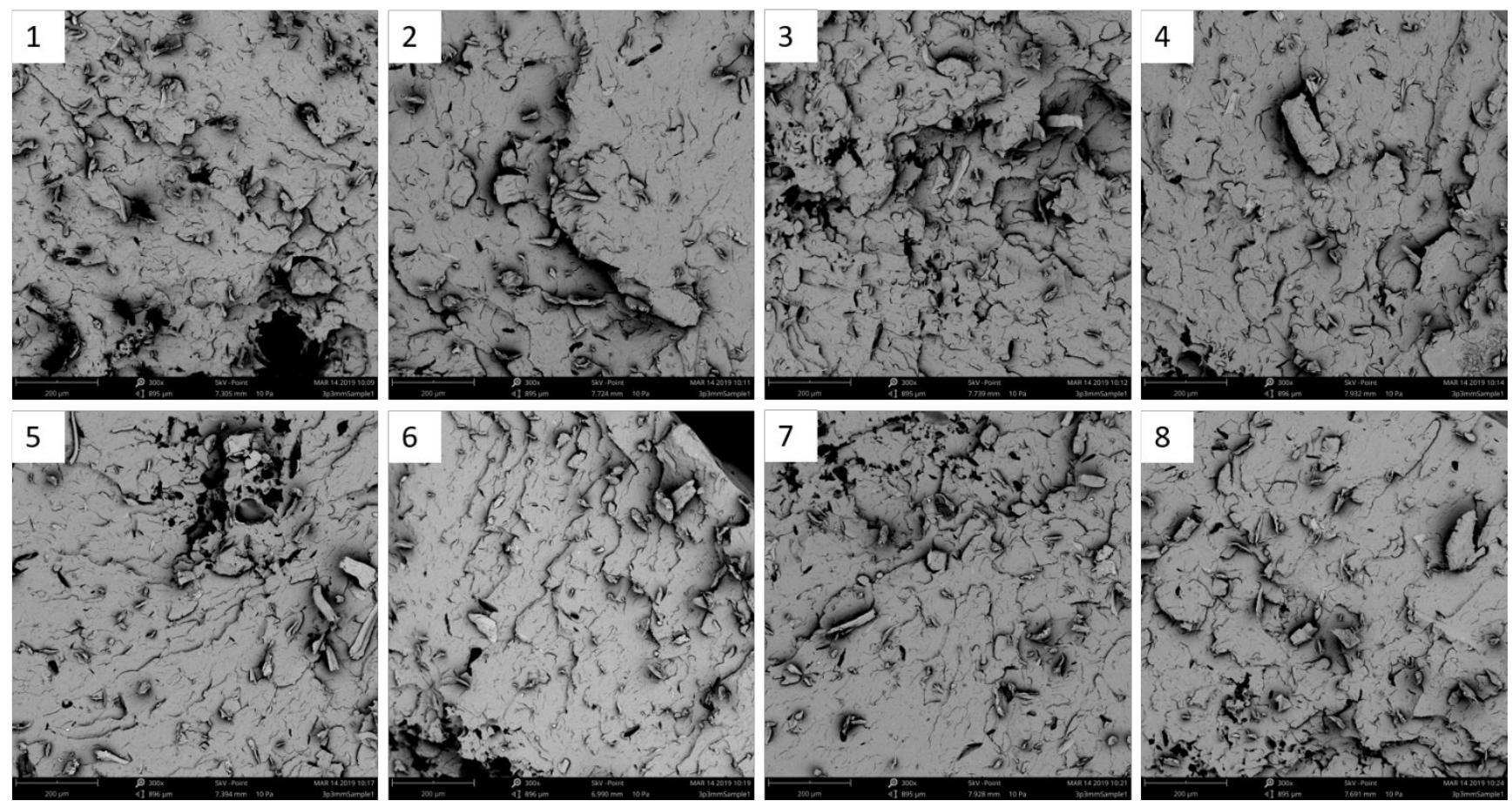

Figure S1. SEM images of cPP/ 10 wt.\% CB filament cross-sections along spool (3 ft. apart)
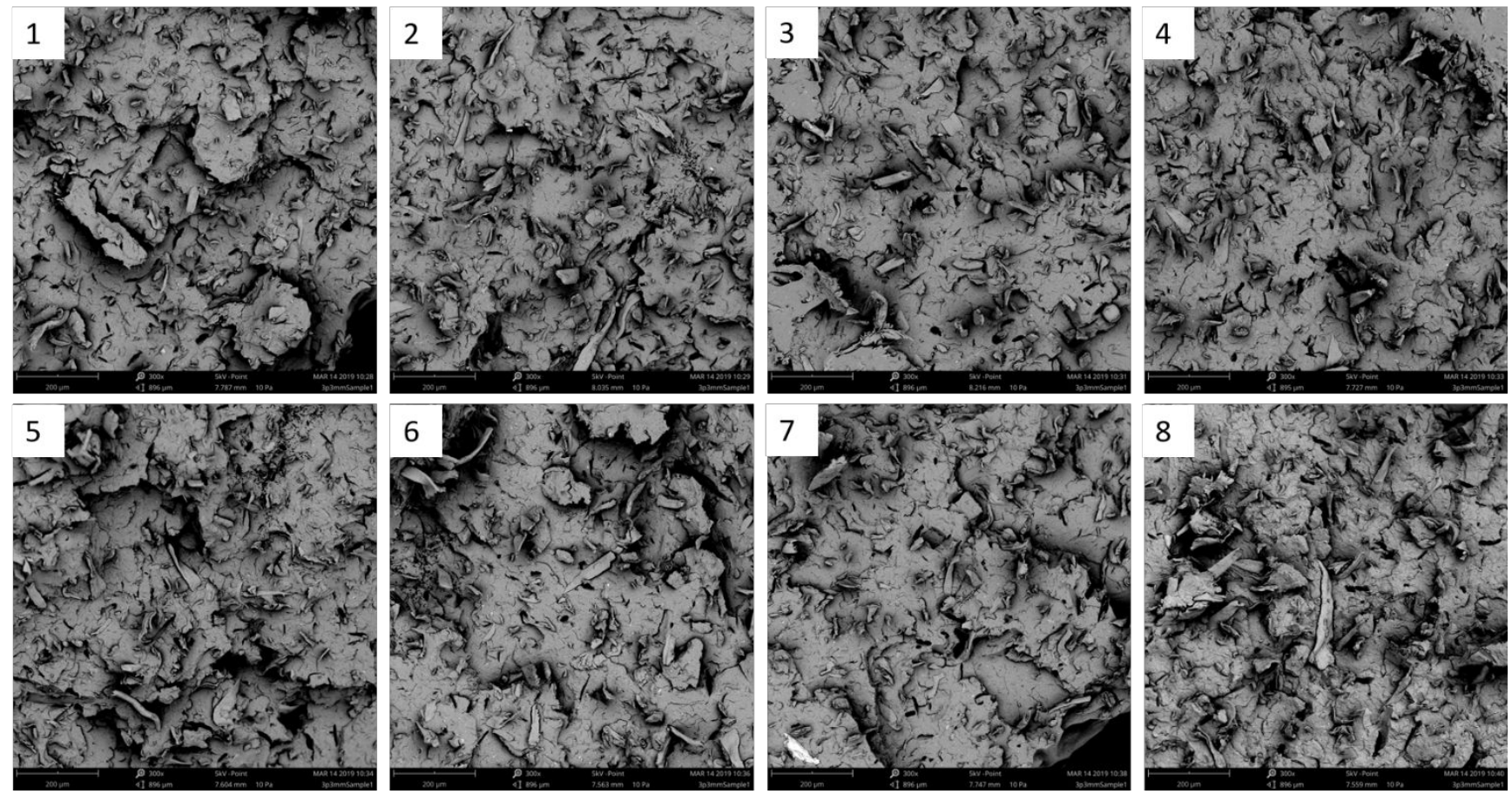

Figure S2. SEM images of rPP/ 10 wt.\% CB filament cross-sections along spool (3 ft. apart) 

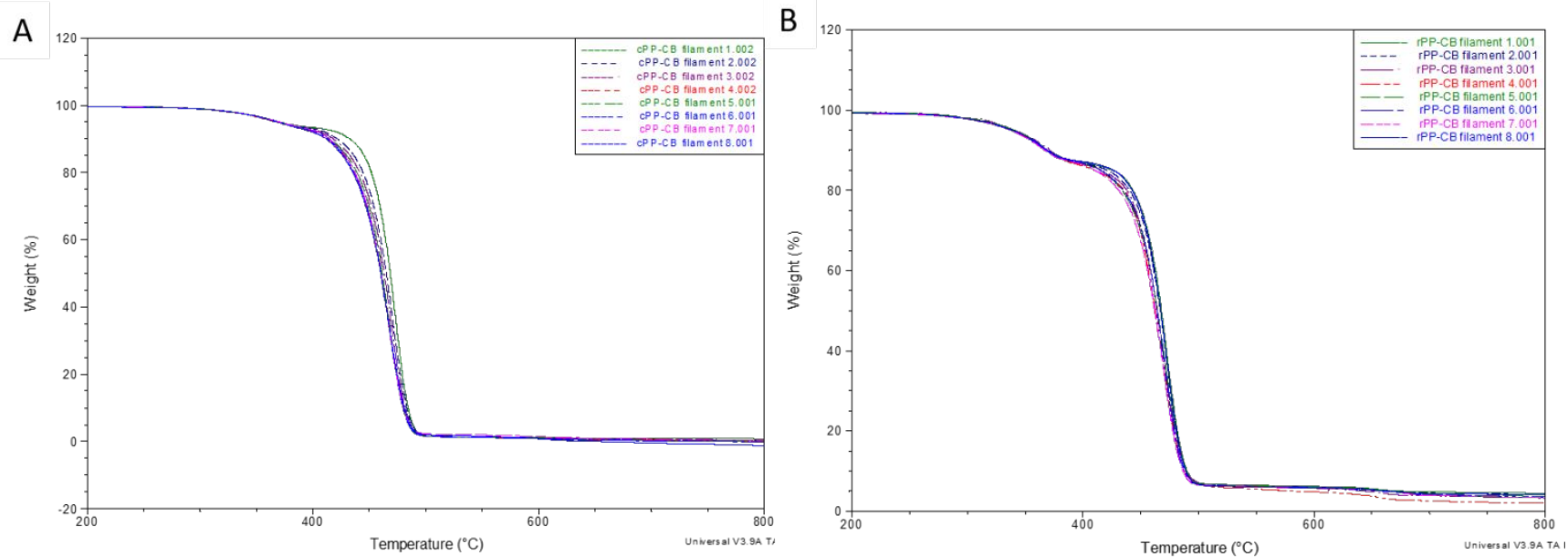

Figure S3. TGA of PP/10 wt.\% CB filament along spool (3 ft. apart). (A) cPP, (B) rPP.
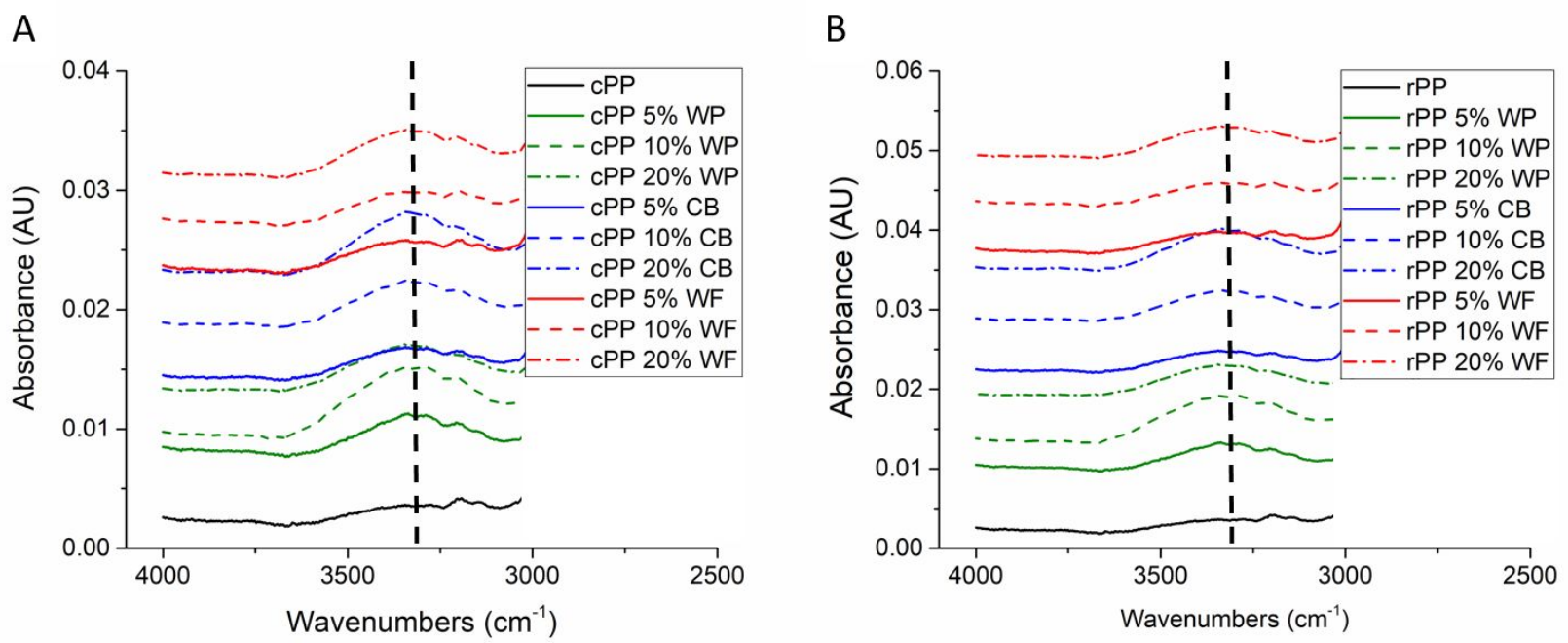

Figure S4. FTIR is printed PP/cellulose (A) cPP, (B) rPP. 
A

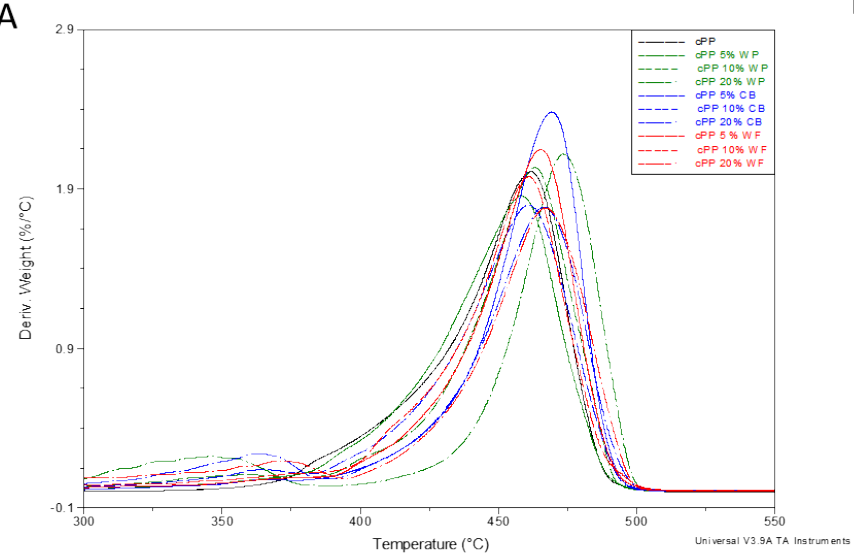

B

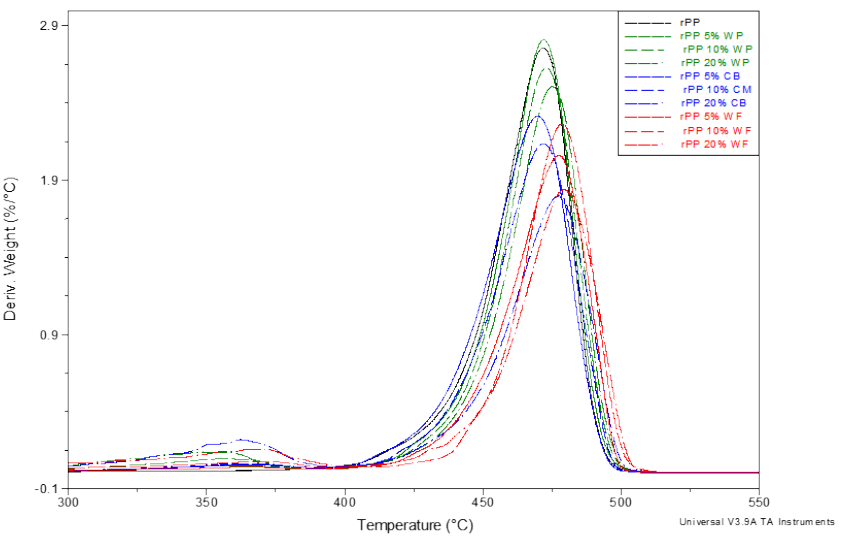

Figure S5. TGA derivative weight percent printed PP/cellulose $(\mathrm{A}) \mathrm{cPP},(\mathrm{B}) \mathrm{rPP}$. Green curves $=$ paper, blue curves $=$ cardboard, red curves $=$ wood flour.
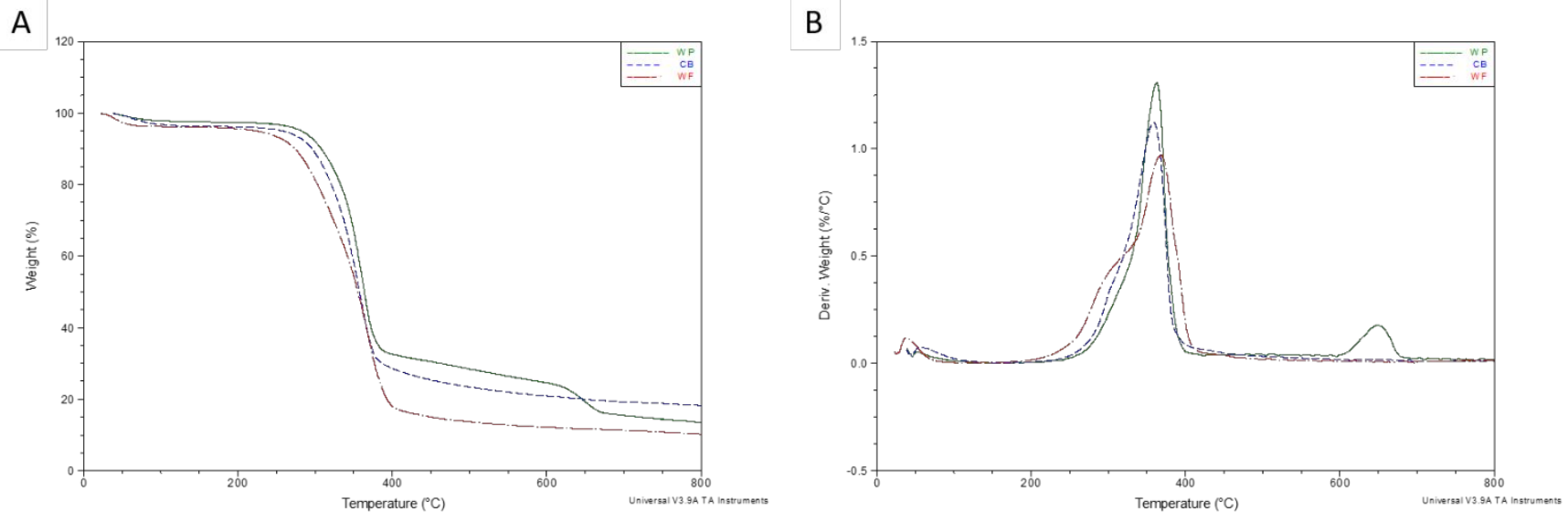

Figure S6. TGA of cellulose fillers (A) Weight loss, (B) Derivative weight. Green curve = paper, blue curve $=$ cardboard, red curve $=$ wood flour.

Table S1. TGA weight percent remaining

\begin{tabular}{|c|c|c|}
\hline Sample & $\begin{array}{l}\text { TGA* } \\
\left(\text { Wt. } \% \text { at } 500{ }^{\circ} \mathrm{C}\right)\end{array}$ & $\begin{array}{l}\text { TGA* } \\
\left(\text { Expected Wt. } \% \text { at } 500{ }^{\circ} \mathrm{C}\right)\end{array}$ \\
\hline rPP & 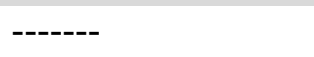 & 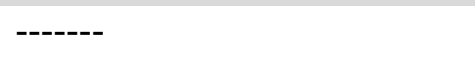 \\
\hline rPP 5\% WP & 0.5 & 1.4 \\
\hline rPP $10 \% \mathrm{WP}$ & 0.9 & 2.8 \\
\hline rPP 20\% WP & 2.5 & 5.7 \\
\hline rPP 5\% CB & 4.9 & 1.2 \\
\hline $\mathrm{rPP} 10 \% \mathrm{CB}$ & 11.5 & 2.3 \\
\hline
\end{tabular}




$\begin{array}{lll}\text { rPP 20\% CB } & 15.6 & 4.6 \\ \text { rPP 5\% WF } & 19.4 & 0.7 \\ \text { rPP 10\% WF } & 16.8 & 1.4 \\ \text { rPP 20\% WF } & 16.8 & 2.7 \\ \text { cPP } & & \\ \text { cPP 5\% WP } & ----- & ----- \\ \text { cPP 10\% WP } & 1.5 & 1.41 \\ \text { cPP 20\% WP } & 2.7 & 2.8 \\ \text { cPP 5\% CB } & 0.5 & 5.7 \\ \text { cPP 10\% CB } & 2.1 & 1.2 \\ \text { cPP 20\% CB } & 3.8 & 2.3 \\ \text { cPP 5\% WF } & 1.3 & 4.6 \\ \text { cPP 10\% WF } & 1.5 & 0.7 \\ \text { cPP 20\% WF } & 3.5 & 1.4 \\ \text { WP } & 28.3 & 2.7 \\ \text { CB } & 23.2 & ----- \\ \text { WF } & 13.6 & ----- \\ \text { WF } & \end{array}$

*controls subtracted 


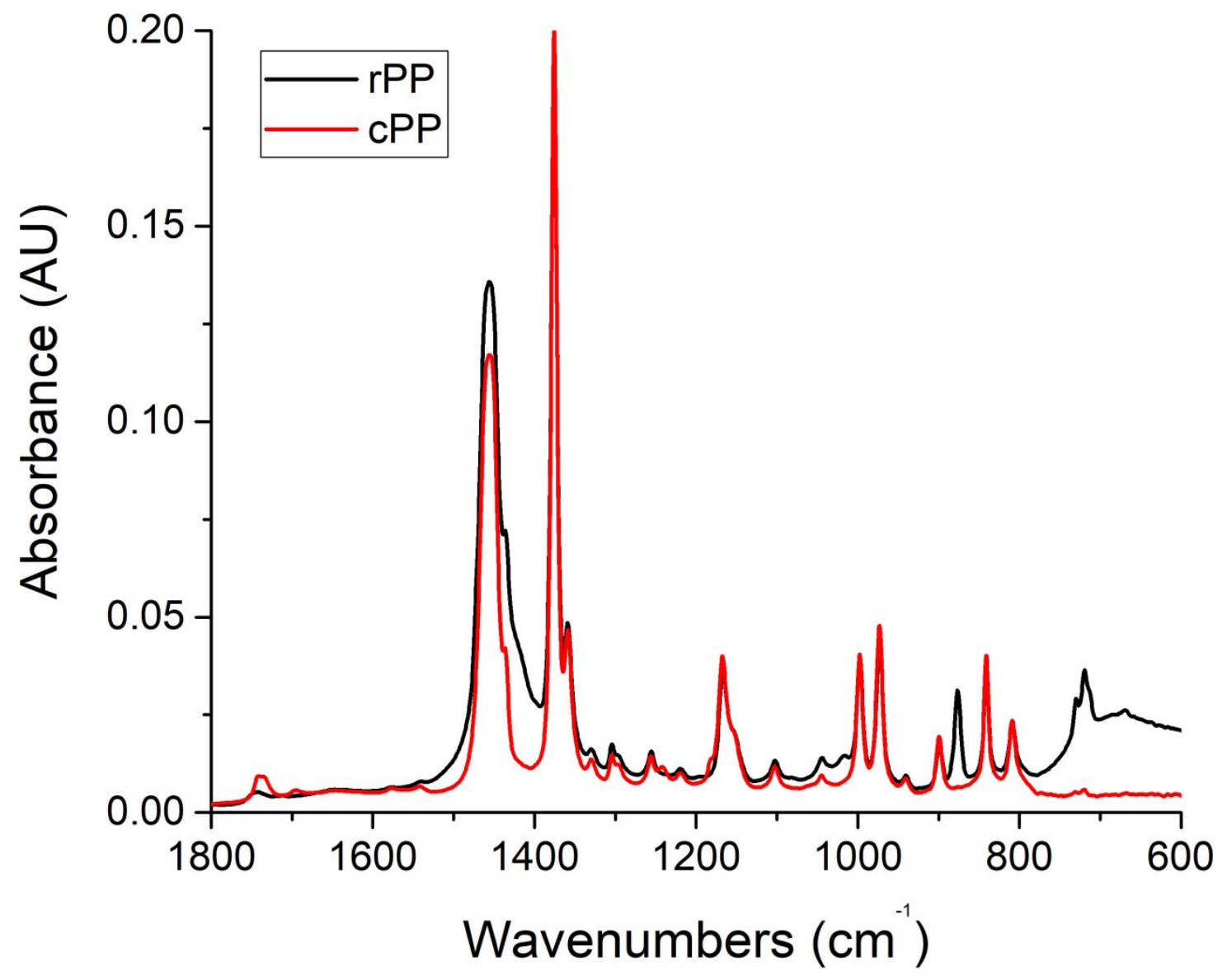

Figure S7. FTIR of rPP and cPP

Table S2. Specific Energy of rPP Composites

\begin{tabular}{|l|l|l|}
\hline Sample & Throughput $(\mathrm{g} / \mathrm{hr})$ & Specific Energy $(\mathrm{kJ} / \mathrm{g})$ \\
\hline $\mathrm{rPP} / 10$ wt.\% WP & 39.4 & 2.7 \\
\hline $\mathrm{rPP} / 10$ wt.\% CB & 39.2 & 2.8 \\
\hline $\mathrm{rPP} / 10$ wt.\% WF & 39.5 & 2.7 \\
\hline
\end{tabular}



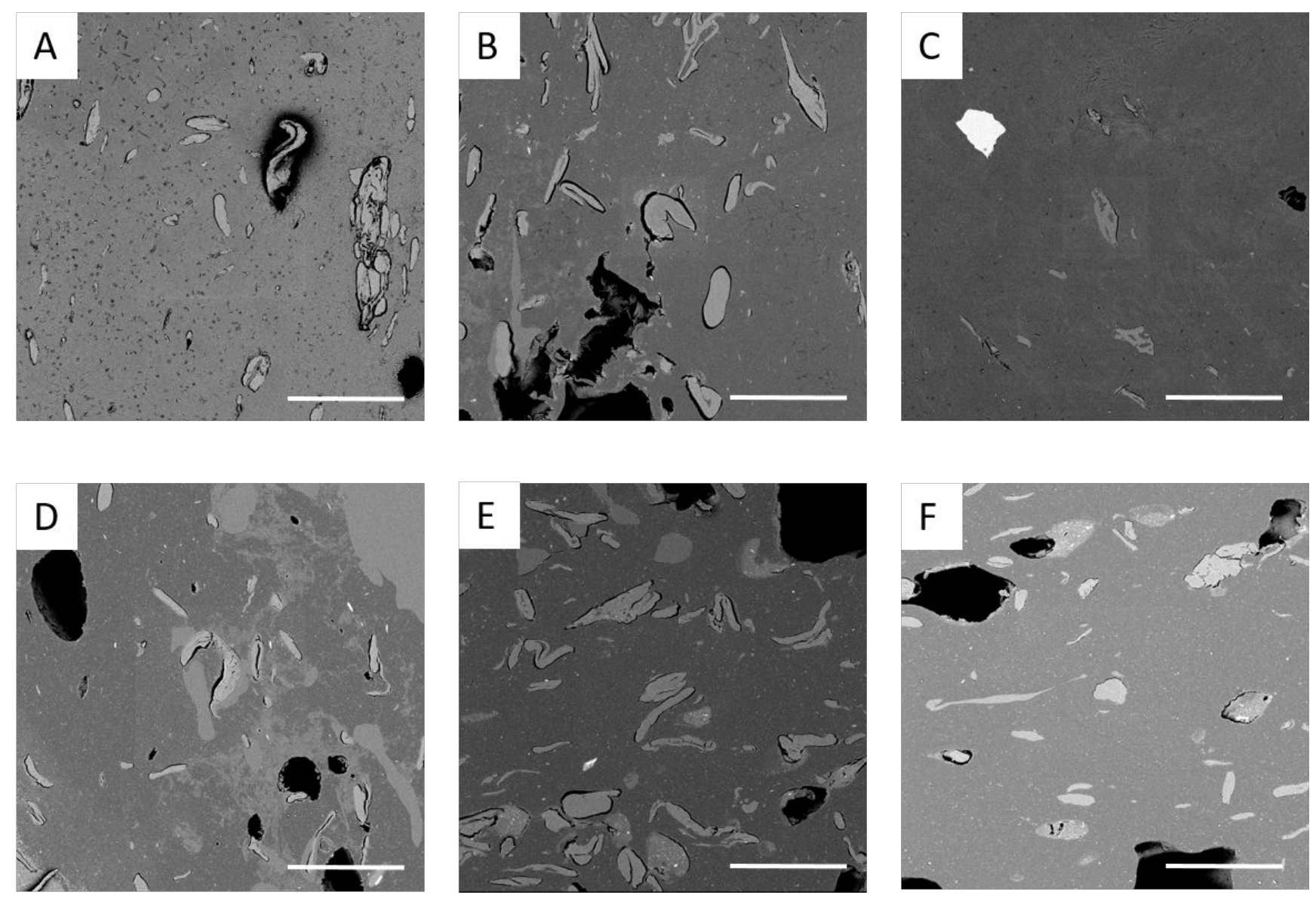

Figure S8. SEM images of polished PP/cellulose fracture surfaces (A) cPP 10\% WP, (B) cPP 10 wt. \% CB, (C) cPP 10 wt. \% WF, (D) rPP 10 wt. \% WP, (E) rPP 10 wt. \% CB, (F) rPP 10 wt. \% WF. Scale bar denotes $80 \mu \mathrm{m}$. 